\title{
Magnetic Order in Li-Mn Spinels
}

\author{
C. B. Azzoni, M. C. Mozzati, A. Paleari ${ }^{a}$, V. Massarotti ${ }^{\mathrm{b}}$, D. Capsoni ${ }^{\mathrm{b}}$ and M. Bini ${ }^{\mathrm{b}}$ \\ INFM Department of Physics “A. Volta” of the University, Via Bassi 6, I-27100 Pavia (Italy) \\ a INFM Department of Materials Science of the University, Via Cozzi 53, I-20126 Milan (Italy) \\ ${ }^{b}$ Department of Physical Chemistry of the University and CSTE-CNR, Viale Taramelli 16, \\ I-27100 Pavia (Italy)
}

Z. Naturforsch. 53a, 693-698 (1998); received April 25, 1998

\begin{abstract}
Magnetic measurements were carried out on different samples of Lithium-Manganese spinel $\mathrm{LiMn}_{2} \mathrm{O}_{4}$, great care having been taken to avoid the presence of spurious magnetic phases, such as $\mathrm{Mn}_{3} \mathrm{O}_{4}$. Susceptibility data, showing deviations from paramagnetic behaviour at about $40 \mathrm{~K}$, were analyzed in terms of local magnetic interactions, taking into account the structural and transport properties of these compounds. The magnetic response of pure and stoichiometric samples suggests that the onset of a longrange magnetic ordering is hindered by the topological frustration of the antiferromagnetic octahedral sublattice of the spinel.
\end{abstract}

Key words: Magnetic Susceptibility; Electron Paramagnetic Resonance; Lithium Manganese Oxides; Lithium Manganese Spinel.

\section{Introduction}

Lithium manganese spinel based compounds have recently received growing interest as to their application in electrochemistry and catalysis and in lithium ion selective detectors. They are also interesting for the coexistence of $\mathrm{Mn}^{4+}$ and $\mathrm{Mn}^{3+}$ valence states [1,2], the latter being a potential Jahn-Teller (JT) ion which may drive structural distortions by cooperative effects $[3,4]$. The occurrence of magnetic ordering in these compounds is strictly related to the effective localization of the electrons introduced by Li substitution; so the study of the magnetic features may be useful for the understanding of the transport features of these compounds.

As regards the magnetic susceptibility $\chi$ of the stoichiometric $\mathrm{LiMn}_{2} \mathrm{O}_{4}$ spinel, different behaviours are reported [1,5-9], showing a sample dependent $\chi$-increase at low temperature. The spread of these results is probably related in part to differences in the preparation methods, i.e. difficulty in obtaining pure and really stoichiometric compounds. However, concerning the magnetic properties of the $\mathrm{LiMn}_{2} \mathrm{O}_{4}$ spinel, some common features may be remarked from the observed high temperature paramagnetic behaviour. The negative Weiss temperature $\theta$ suggests a prevalent antiferromagnetic character of the local magnetic interactions. Moreover, the calculated magnetic moment per $\mathrm{Mn}$ ion $[1,6]$ is consistent

Reprint requests to Prof. C. B. Azzoni; Fax: 0382-507563. with the coexistence of $\mathrm{Mn}^{4+}$ ions $(S=3 / 2)$ with $\mathrm{Mn}^{3+}$ ions in the high spin configuration $(S=2)$, with the fourth electron in a $\mathrm{e}_{g}$ orbital. This electron localization is supported by charge transport measurements [1], since the rather low conductivity $\sigma\left(10^{-4} \Omega^{-1} \mathrm{~cm}^{-1}\right.$ at $\left.300 \mathrm{~K}\right)$ and the weak thermal dependence of the thermopower suggest the presence of an electron hopping mechanism with an activation energy of $0.4 \mathrm{eV}$. Moreover, independent evidence of electron localization in $\mathrm{e}_{g}$ orbitals comes from structural data. In fact, nearly stoichiometric $\mathrm{Li}\left(\mathrm{Li}_{y} \mathrm{Mn}_{2-y}\right) \mathrm{O}_{4}$ compounds, from $\mathrm{Li}$ enrichment of $\mathrm{LiMn}_{2} \mathrm{O}_{4}$ limited in the range $0<y<0.04$, show structural changes with lowering the transition temperature below $280 \mathrm{~K}[3,4]$. These changes were ascribed to the onset of a cooperative JT transition arising from tetragonal elongation of the coordination octahedra around the $\mathrm{Mn}^{3+}$ ions.

This work aims at investigating the magnetic interactions in Lithium-Manganese spinels, by means of susceptibility, magnetization and EPR measurements, in strict connection with structural determinations.

\section{Sample Preparation and Structural Details}

Several samples were synthesized by a solid state reaction from starting mixtures $\mathrm{MnO}-\mathrm{Li}_{2} \mathrm{CO}_{3}$ with 0.333 Lithium cationic fraction. Each mixture was heated 8 hours in air at $1073 \mathrm{~K}$ with heating and cooling rates 


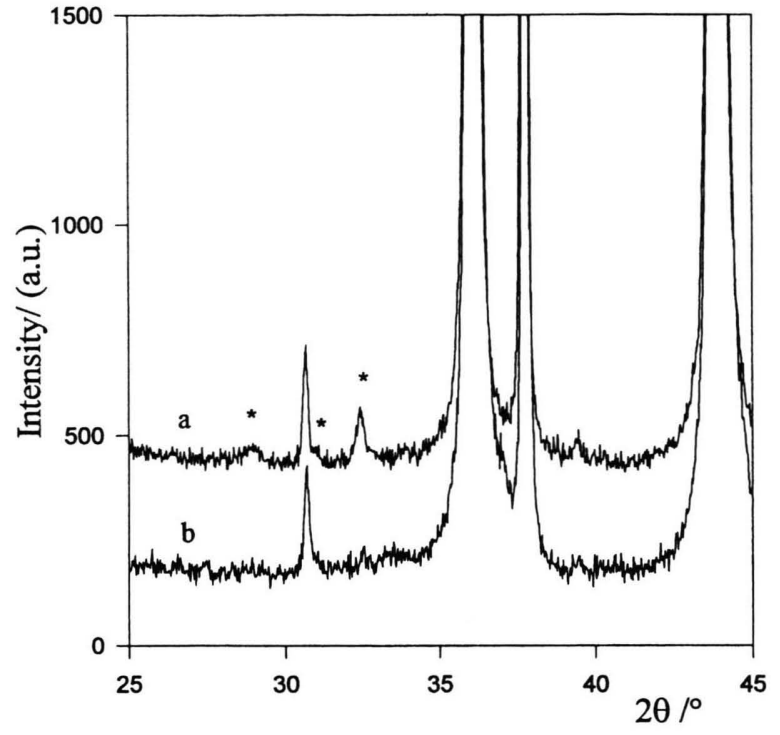

Fig. 1. Comparison of XRD patterns of Lithium-Manganese spinel $a$ and $b$ in a limited $2 \theta$ range: $\mathrm{Mn}_{3} \mathrm{O}_{4}$ peaks in the $a$-sample are marked by stars.

of $5 \mathrm{~K} / \mathrm{min}$ : these will be referred to hereafter as $a$-samples.

The characterization by $x$-ray diffraction of the $a$-samples showed the presence of small amounts of the $\mathrm{Mn}_{3} \mathrm{O}_{4}$ phase, ferrimagnetic at low temperature [10]. Other procedures were also employed for comparison. Then, one $a$-sample was furtherly treated with particular care, being reheated several times at $1073 \mathrm{~K}$ and ground, to assure the complete transformation of the impurity phase, so that its magnetic properties were not affected by the presence of $\mathrm{Mn}_{3} \mathrm{O}_{4}$ ( $b$-sample). Indeed the characterization by $\mathrm{x}$-ray diffraction of the $b$-sample showed the presence of the spinel phase alone, as proved by the pattern comparison (Figure 1). Both spinel lines and the most important peaks of the $\mathrm{Mn}_{3} \mathrm{O}_{4}$ phase (marked by stars) are present in the $a$-sample, but the latter are absent in the $b$-one.

Besides, the JT transition temperature $T_{\mathrm{JT}}$ is a sensitive indicator of the spinel stoichiometry. Actually, $T_{\mathrm{JT}}$ changes from $280 \mathrm{~K}$ at $y=0$ to $200 \mathrm{~K}$ at $y=0.039$ [4], (where $y$ is the Lithium stoichiometry deviation) and at the same time the measured enthalpy of the transition gradually decreases. Our samples, with distinct $\chi$ and $\sigma$ anomalies at $280 \mathrm{~K}$, related to the JT transition [1, 5], may be considered stoichiometric. The value of the lattice parameter (for $a$-sample $a=0.8243(1) \mathrm{nm}$; for $b$-sample $a=0.8239(1) \mathrm{nm}$ ) is also pertinent to the stoi-

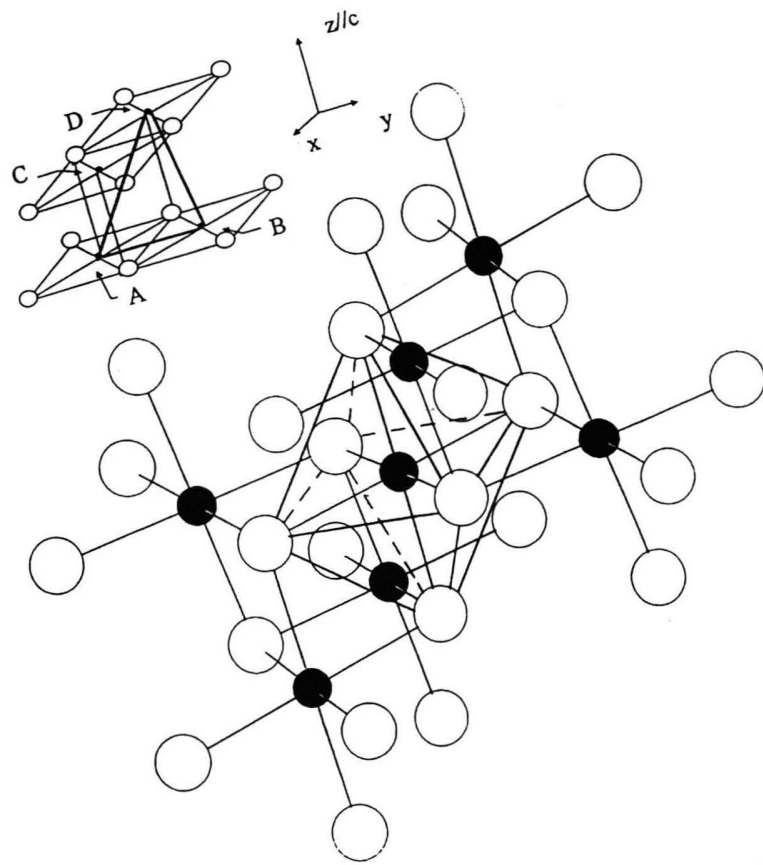

Fig. 2. Coordination oxygen (open circles) octahedra around $\mathrm{Mn}$ sites (filled circles) in $\mathrm{LiMn}_{2} \mathrm{O}_{4}$. Six octahedra nearest neighbours to the central one are shown. The inset schematically displays the cluster considered to describe the magnetic interactions and the triangular network of the Mn spinel sublattice.

chiometric sample as expected from the comparison with literature values of $0.8246 \mathrm{~nm}$ [11].

In order to discuss the physical properties of these compounds, it is important to describe the peculiar Mn sites and the pertinent $\mathrm{Mn}-\mathrm{O}-\mathrm{Mn}$ array. $\mathrm{LiMn}_{2} \mathrm{O}_{4}$ is cubic, spinel type $(\mathrm{Fd} 3 \mathrm{~m})$ with atomic positions deduced from [11]. More details about the structure can be given by referring to the coordination octahedra of oxygen ions around the Mn site, as shown in Figure 2. The average Mn-O distance is $0.1983 \mathrm{~nm}$. Six neighboring octahedra are centered on other $\mathrm{Mn}$ ions and share the edges with the central one. The Mn-O-Mn angles are about $95^{\circ}$ while the O-Mn-O angles are about $85^{\circ}$.

\section{Experimental Procedure}

Static magnetic susceptibility and magnetization measurements were carried out from $300 \mathrm{~K}$ down to $4 \mathrm{~K}$, in magnetic fields ranging between 50 and $4500 \mathrm{G}$, by using a Faraday balance susceptometer with a continu- 
ous-flow cryogenic apparatus. The final accuracy of the mass susceptibility $\left(\chi_{m}\right)$ data results in an error of a few $\%$. The magnetic field dependence was analyzed by changing the field intensity at fixed temperatures.

EPR measurements were carried out by using a Bruker spectrometer at $9.12 \mathrm{GHz}$ between 120 and $473 \mathrm{~K}$.

Diffraction data were obtained by a Philips PW1710 powder diffractometer equipped with a Philips PW1050 vertical goniometer. Use was made of the $\mathrm{CuK} \alpha$ radiation $\left(\mathrm{K} \alpha_{1}=1.54056 \AA ; \mathrm{K} \alpha_{2}=1.5443 \AA\right)$ by means of a graphite monochromator. Patterns were collected in the angular range $15^{\circ}<2 \theta<130^{\circ}$ in the step scan mode (step width $0.025^{\circ}$; counting time $10 \mathrm{~s}$ ). Further details and computational procedure have been given in $[1,2]$.

\section{Results}

In Fig. 3 the $\chi_{\mathrm{m}}(T)$ and $\chi_{\mathrm{m}}^{-1}(T)$ curves of the $b$-sample are reported down to $4 \mathrm{~K}$. The change in the slope of $1 / \chi_{\mathrm{m}}$ at about $40 \mathrm{~K}$ might suggest the onset of a magnetic ordering. The paramagnetic region is not characterized by a well defined Weiss temperature $\theta$ because for $T>280 \mathrm{~K}$ a pronounced deviation from linearity is observable. However, a negative temperature intercept $\theta \cong-320 \mathrm{~K}$ may be argued. This value is confirmed in [8], where the $\chi_{\mathrm{m}}^{-1}(T)$ curve is reported up to $800 \mathrm{~K}$. For the same sample, data of the mass magnetization $M$ vs. applied field at different temperatures are reported in Figure 4. $M$ follows linear behaviours strictly proportional to the field intensity, also below $40 \mathrm{~K}$, as in non magnetically ordered compounds. The extrapolated zero field $M(0)$ val-

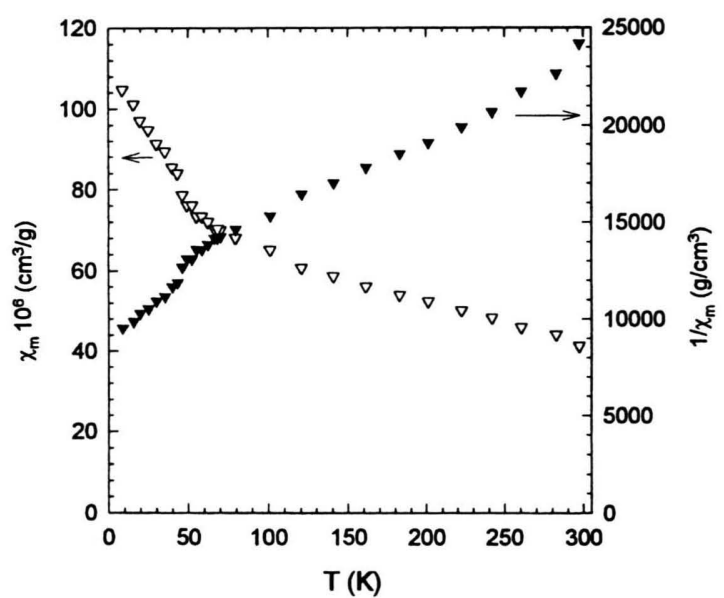

Fig. 3. Temperature dependence of $\chi_{\mathrm{m}}$ (open marks) and $\chi_{\mathrm{m}}^{-1}$ (filled marks) of the $b$-sample. ues are reported in Fig. 5, as functions of the temperature for the $b$ - and $a$-sample. Only the $a$-sample shows a weak net resultant magnetic moment, due to the presence of few $\%$ of $\mathrm{Mn}_{3} \mathrm{O}_{4}$ phase $(\approx 1.5 \%$ ) (see the inset of Fig. 5), while the $b$-sample shows a negligible $M(0)$ value. This behaviour of $M(0)$ confirms the lack of $\mathrm{Mn}_{3} \mathrm{O}_{4}$

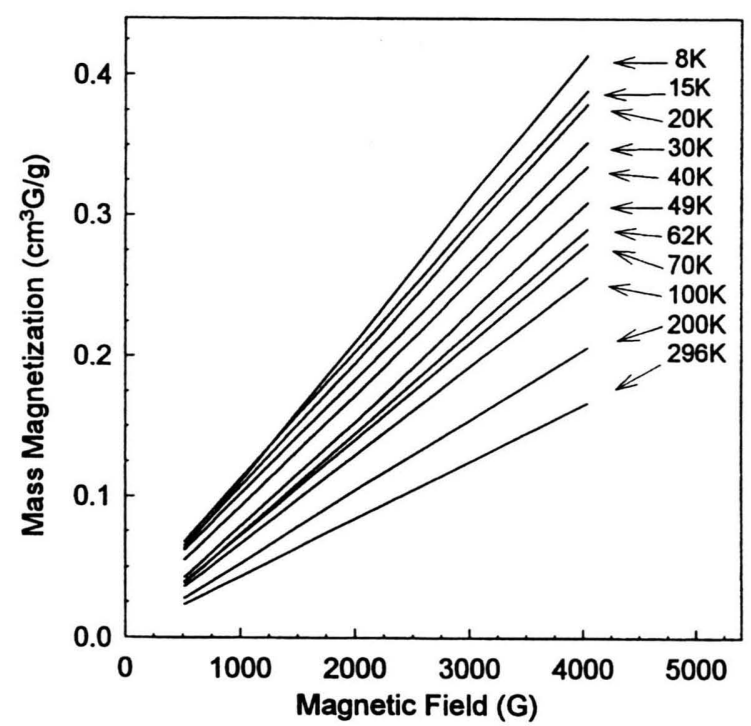

Fig. 4. Mass magnetization curves at different temperatures of the $b$-sample.

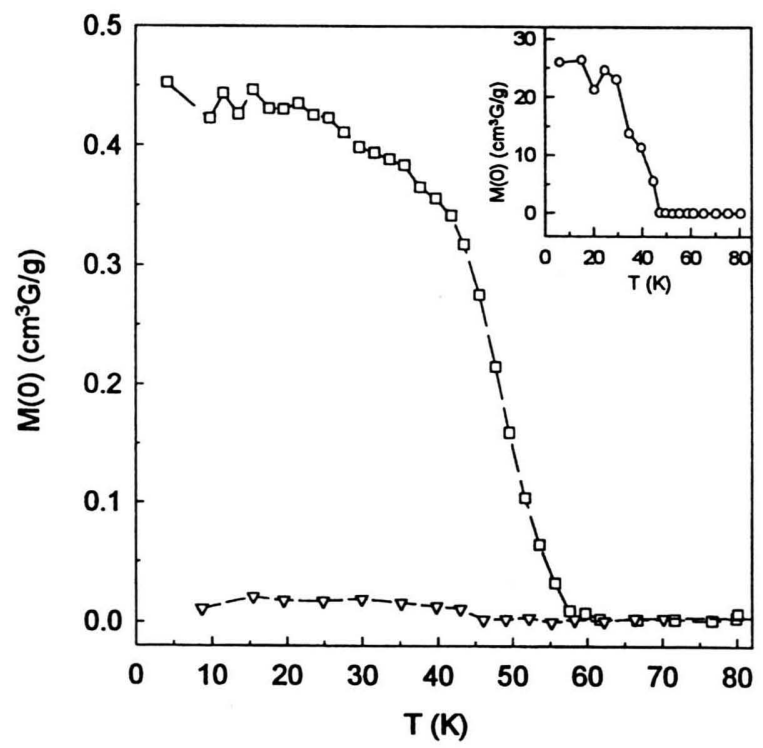

Fig. 5. Mass magnetization curves vs. Temperature of the $b$-sample (triangles), of an $a$-sample (squares) and of $\mathrm{Mn}_{3} \mathrm{O}_{4}$ (inset). 


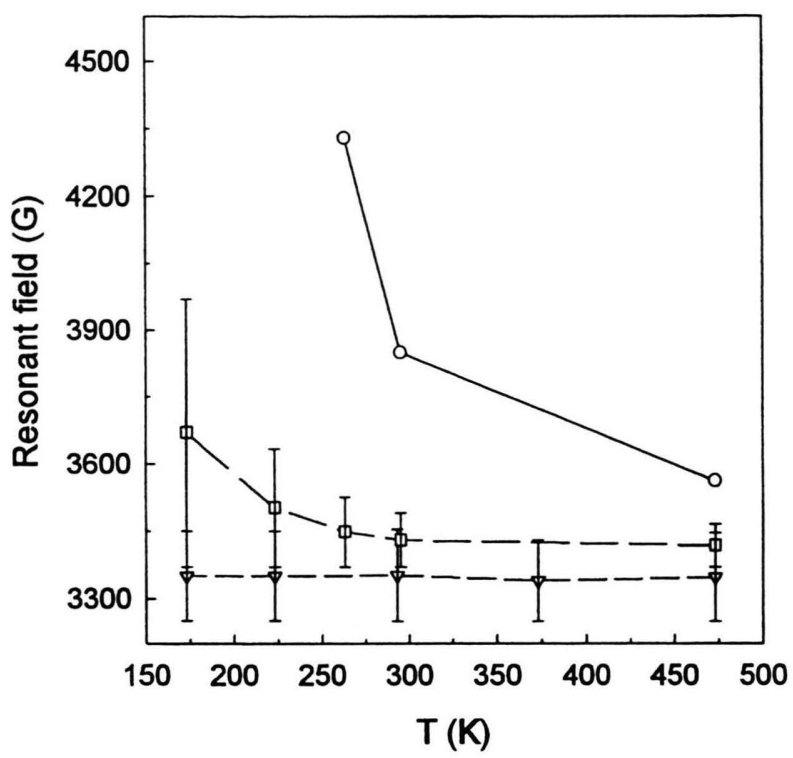

Fig. 6. Resonant field vs. Temperature of the EPR signal for the $b$-sample (triangles), an $a$-sample (squares) and $\mathrm{Mn}_{3} \mathrm{O}_{4}$ (circles).

phase in the reheated spinel. This assertion is also supported by Fig. 6, reporting the different behaviour of the resonant field (RF) of EPR spectra for spinel samples and $\mathrm{Mn}_{3} \mathrm{O}_{4}$ : only the $b$-sample shows a RF which does not depend on the temperature while the $\mathrm{RF}$ behaviour of the $a$-sample is similar to that observed in $\mathrm{Mn}_{3} \mathrm{O}_{4}$, although less pronounced.

\section{Discussion}

At a first glance, the $1 / \chi_{\mathrm{m}}$ curve in the spinel (Fig. 3) suggests an antiferromagnetic-like behaviour below $40 \mathrm{~K}$, as also reported in [8]. This is also consistent with the negative $\theta$ of the paramagnetic phase, i.e. with a prevalent AF character of the local magnetic interactions. Nevertheless, we cannot observe a clear-cut magnetic transition but only a deviation from the paramagnetic regime, suggesting that the onset of a true magnetic ordered phase is hindered.

Consideration of structural and transport features of these materials may clarify the origin of this behaviour. The electronic conduction is characterized by the hopping of electrons through $\mathrm{Mn}^{4+}$ ions, and this is consistent with the value of the conductivity at high temperature (a few $\Omega^{-1} \mathrm{~cm}^{-1}$ at about $1000 \mathrm{~K}$ ) [1]. Nevertheless, the hopping conductivity at room temperature is very low, and the electrons are to be considered effectively localized in $\mathrm{Mn}^{4+}$ to form $\mathrm{Mn}^{3+}$ sites. This localization is evidenced by the JT distortion, which pertains to the $\mathrm{d}^{4}$ configuration of the $\mathrm{Mn}^{3+}$ ions. The discussion of the magnetic properties will now be done, starting from the anal$y$ sis of the possible interaction pictures in the local environments of the Mn sites, in order to permit a comparison with the experimental data.

We remark that the cubic to tetragonal transition at $280 \mathrm{~K}$ of the $\mathrm{Mn}^{3+} \mathrm{O}_{6}$ octahedra splits the energy levels of the $\mathrm{e}_{g}$ orbitals. By performing crystal field calculations on the octahedral coordination, according to the distortion $c / a=1.011$ [3], we obtained an energy splitting of $0.08 \mathrm{eV}$ between the $\mathrm{d}_{3 z 2-r 2}$ and the $\mathrm{d}_{x 2-y 2}$ states, with the former at lower energy. In the case of $\mathrm{Mn}^{3+}$ in high spin configuration, the $\mathrm{d}_{3 z 2-r 2}$ state is half-filled while the $d_{x 2-y 2}$ one is empty. These orbitals should give the main contribution to $90^{\circ}$ superexchange interactions via oxygen orbitals. Even though in the stoichiometric spinel each $\mathrm{Mn}$ ion has six next neighbours, at a mean distance of $0.2916 \mathrm{~nm}$, it is convenient to consider the possible interactions within the cluster, or magnetic and structural unit, reported in the inset of Fig. 2, which generates the spinel lattice by translation.

The following cases regard superexchange interactions mediated by a single oxygen orbital: between $A$ and $B$ ions (see Fig. 2), two ferromagnetic (F) interactions with $(\sigma \pi)$ character from the overlap between $\mathrm{d}_{x 2-y 2}$ and $\mathrm{d}_{x y}$ and two antiferromagnetic (AF) $(\pi \pi)$ interactions from the overlap of $\mathrm{d}_{y z}$ and $\mathrm{d}_{x z}$. Between $\mathrm{A}$ and C, D ions, two $\mathrm{F}$ interactions occur with $(\sigma \pi)$ character between $\mathrm{d}_{x 2-y 2}$ and $\mathrm{d}_{x z}\left(\right.$ or $\left.\mathrm{d}_{y z}\right)$ and two $\mathrm{AF}(\pi \pi)$ interactions between $d_{x y}$ and $d_{x z}\left(\right.$ or $d_{y z}$ ) (Fig. 7 (I, II)). This process should not be likely, owing to the high energy of the excited levels due to Coulomb interaction. Moreover, all these superexchange contributions should be rather small involving weak $\pi$ overlap between $\mathrm{p}$ and $\mathrm{d}$ orbitals.

Superexchange interactions via two orthogonal oxygen orbitals are also possible: one $\mathrm{F}(\sigma \sigma)$ between $\mathrm{A}$ and $\mathrm{B}$ ions, and another $\mathrm{F}(\sigma \sigma)$ between $\mathrm{A}$ and $\mathrm{C}$, D ions arising from the overlap of $\mathrm{e}_{g}$ orbitals with $\mathrm{p}$ oxygen orbitals taking into account the Hund rule for the spins of the two half-filled orbitals of the oxygen (Fig. 7 (III)). The full $\sigma$ character of these interactions suggests that these should prevail over the others, also because they do not call for orbitals already involved into direct interactions.

In fact, since the coordination octahedra share a common edge, direct magnetic interactions take place between Mn ions through half-filled $\mathrm{t}_{2 g}$ orbitals [12]. These interactions are $\mathrm{AF}$ and the more intense the smaller the 
I)

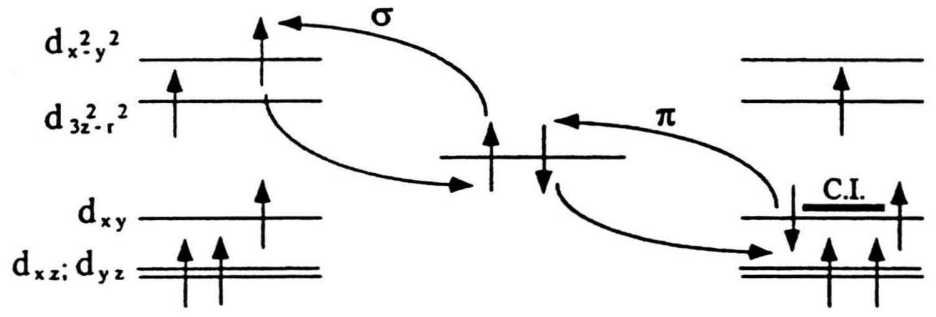

II)

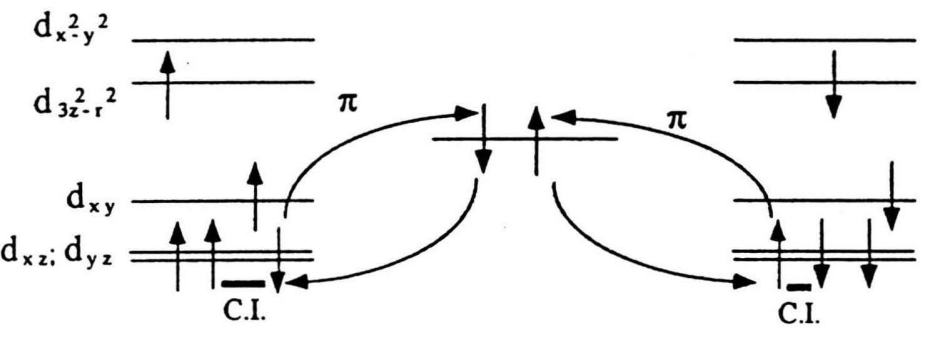

III)

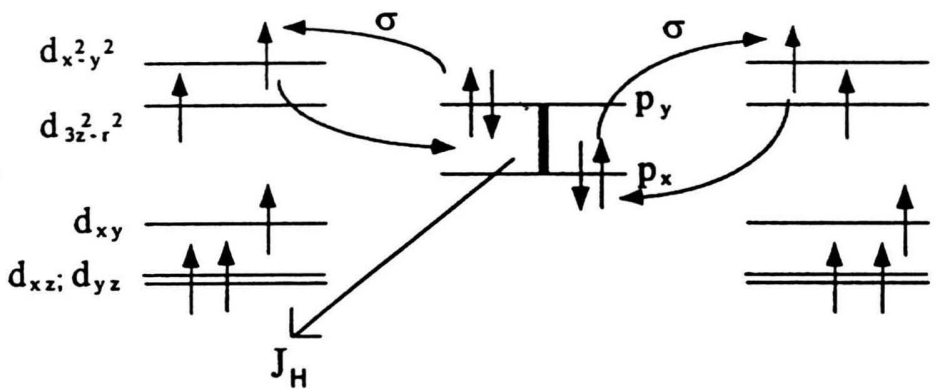

Fig. 7. Magnetic interaction mechanisms in $\mathrm{Mn}^{3+}-\mathrm{O}-\mathrm{Mn}^{3+}$ complexes via one oxygen p-orbital (I and II) and two oxygen p-orbitals (III): (I) ferromagnetic interaction $\mathrm{d}_{x 2-y 2}-\mathrm{p}-\mathrm{d}_{x y}$; (II) antiferromagnetic interaction $\mathrm{d}_{x y}-\mathrm{p}-\mathrm{d}_{z x}$; (III) ferromagnetic interaction $\mathrm{d}_{x 2-y 2}-\mathrm{p}_{y}: \mathrm{p}_{x}-\mathrm{d}_{x 2-y 2}$. The same mechanisms hold in the cases of $\mathrm{Mn}^{3+}-\mathrm{O}-\mathrm{Mn}^{4+}$ and $\mathrm{Mn}^{4+}-\mathrm{O}-\mathrm{Mn}^{4+}$ interactions, provided that the $d_{3 z 2-r 2}$ orbital of one or both cations, respectively, is empty. The Hund's rule interaction is indicated by $J_{\mathrm{H}}$, and the Coulomb interaction by C.I. distance between the cations. A direct AF interaction will be active between $d^{3}-d^{3}$ or $d^{4}-d^{4}$ and $d^{3}-d^{4}$ ion couples through the overlap of $\mathrm{d}_{x y}, \mathrm{~d}_{x z}$ and $\mathrm{d}_{y z}$ orbitals.

In summary, the $\mathrm{A}-\mathrm{C}, \mathrm{D}$ interaction results from two direct $\mathrm{AF}$ and a superexchange $\mathrm{F}$ one, while the resulting $\mathrm{A}-\mathrm{B}$ interaction arises from a direct $\mathrm{AF}$ interaction and a superexchange one comprising $\mathrm{F}$ contributions. Both could be in principle either $\mathrm{F}$ or $\mathrm{AF}$, but experimental data and transport and structural features of these compounds (similarly to other insulator oxides with halffilled $t_{2 g}$ orbitals $[12,13]$ ) suggest that the direct interactions play the relevant role in determining the magnetic properties, and the resulting local interactions should be AF.

Consideration of the local geometry in the spinel lattice (see Fig. 2) shows that magnetic ions lie in the sites of a triangular network of the transition cation sublattice. No magnetic long-range ordering of spins is possible, because of the frustration of the AF interaction between some ion pairs. This is a typical situation occurring in an octahedral AF sublattice giving rise to a spin-glass type behaviour, according to [9]. By contrast, more evident deviations or magnetic transitions observed in the same range of temperature in other spinel samples (as in our 
$a$-samples) are to be attributed to spurious magnetic phases, as ferrimagnetic $\mathrm{Mn}_{3} \mathrm{O}_{4}$.

\section{Conclusion}

Susceptibility and magnetization measurements, compared with data from conductivity [1], thermal analysis $[3,4]$, and $\mathrm{x}$-ray powder diffraction [2] measurements, allowed us to put forward a correlation among electron localization, onset of cooperative JT effect and magnetic features below $40 \mathrm{~K}$ in the $\mathrm{LiMn}_{2} \mathrm{O}_{4}$ spinel.

[1] V. Massarotti, D. Capsoni, M. Bini, G. Chiodelli, C. B. Azzoni, M. C. Mozzati, and A. Paleari, J. Solid State Chem. 131, 94 (1997).

[2] V. Massarotti, D. Capsoni, M. Bini, C. B. Azzoni, and A. Paleari, J. Solid State Chem. 128, 80 (1997).

[3] A. Yamada and M. Tanaka, Mat. Res. Bull. 30,715 (1995).

[4] A. Yamada, J. Solid State Chem. 122, 160 (1996).

[5] J. Sugiyama, T. Tamura, and H. Yamauchi, J. Phys.: Condensed Matter 7, 9755 (1995).

[6] C. Masquelier, M. Tabuchi, K. Ado, R. Kanno, Y. Kobayashi, Y. Maki, O. Nakamura, and J. B. Goodenough, J. Solid State Chem. 123, 255 (1996).

[7] N. Kumagai, T. Fujiwara, K. Tanno, and T. Horiba, J. Electrochem. Soc. 143, 1007 (1996).
The magnetic response of pure and stoichiometric samples was detailed by considering the possible local magnetic interactions consistent with the structure. This analysis, together with geometrical arguments on the magnetic sublattice, suggests that the onset of a long-range magnetic ordering is hindered by the topological frustration of the antiferromagnetic octahedral sublattice of the spinel.

\section{Acknowledgements}

This work has been partially supported by Consorzio per i Sistemi a Grande Interfase (CSGI) and by Progetto Fìnalizzato MSTA II-Accumulo di Energia.

[8] J. Sugiyama, T. Hioki, S. Noda, and M. Kontani, J. Phys. Soc. Japan 66-1, 1187 (1997).

[9] P.Endres, B. Fuchs, S. Kemmler-Sack, K. Brandt, G. FaustBecker, and H.-W. Praas, Solid State Ionics 89, 221 (1996).

[10] B. Boucher, R. Buhl, and M. Perrin, J. Phys. Chem. Solids 32, 2429 (1971).

[11] D. G. Wickham and W. J. Croft, J. Phys. Chem. Solids 7, 351 (1958).

[12] J. B. Goodenough, Phys. Rev. 117, 1442 (1960).

[13] V. Massarotti, D. Capsoni, M. Bini, C. B. Azzoni, M. C. Mozzati, and A. Paleari, Z. Naturforsch. 53a, 150 (1998). 